Article

\title{
Novel Biochip Platform for Nucleic Acid Analysis
}

\section{Salvatore Pernagallo ${ }^{1, \dagger}$, Giorgio Ventimiglia ${ }^{2, *, \dagger}$, Claudia Cavalluzzo ${ }^{1}$, Enrico Alessi ${ }^{2}$, Hugh Ilyine ${ }^{1}$, Mark Bradley ${ }^{3}$ and Juan J. Diaz-Mochon ${ }^{1}$ *}

1 DestiNA Genomics Ltd., West Mains Road, Edinburgh EH9 3JJ, UK;

E-Mails: salvatore.pernagallo@destinagenomics.com (S.P.); claudia@destinagenomics.com (C.C.); hugh@destinagenomics.com (H.I.)

2 STMicroelectronics, ANALOG, MEMS and SENSORS Group, Healthcare BDU, Stradale Primosole 50, Catania 95121, Italy; E-Mail: enrico.alessi@ st.com

3 School of Chemistry, University of Edinburgh, West Mains Road, Edinburgh EH9 3JJ, UK;

E-Mail: mark.bradley@ed.ac.uk

$\dagger$ These authors contributed equally to this work.

* Authors to whom correspondence should be addressed; E-Mails: giorgio.ventimiglia @ st.com (G.V.); juan@destinagenomics.com (J.J.D.-M.); Tel.: +39-095-740-4344 (G.V.); +44-0-131-650-4821 (J.J.D.-M.).

Received: 25 April 2012; in revised form: 21 May 2012 / Accepted: 31 May 2012 /

Published: 11 June 2012

\begin{abstract}
This manuscript describes the use of a novel biochip platform for the rapid analysis/identification of nucleic acids, including DNA and microRNAs, with very high specificity. This approach combines a unique dynamic chemistry approach for nucleic acid testing and analysis developed by DestiNA Genomics with the STMicroelectronics In-Check platform, which comprises two microfluidic optimized and independent PCR reaction chambers, and a sequential microarray area for nucleic acid capture and identification by fluorescence. With its compact bench-top "footprint" requiring only a single technician to operate, the biochip system promises to transform and expand routine clinical diagnostic testing and screening for genetic diseases, cancers, drug toxicology and heart disease, as well as employment in the emerging companion diagnostics market.
\end{abstract}


Keywords: Lab-on-Chip (LoC); microarray; nucleic acid; dynamic chemistry; polymerase chain reaction (PCR); peptide nucleic acid (PNA); microfluidic; single nucleotide polymorphism (SNP); microRNA-122 (miRNA122); mengo virus (MGV); nucleic acid test (NAT); point-of-care (POC)

\section{Introduction}

The development of rapid diagnostic device platforms is one of the major scientific aims in the world of life-science research, drug discovery, medical diagnostics and biotechnology [1-7]. Several point-of-care (POC) diagnostic platforms have been launched in the past few years with some approved by the Food and Drug Administration (FDA) for in vitro diagnostic (IVD) applications [8-10]. Emerging nucleic acid test (NAT) technologies have allowed the development of applications for genotyping [single nucleotide polymorphisms (SNPs) and indel identification], epigenetic studies, array comparative genome hybridisation $(\mathrm{aCGH})$, pre-natal screening, and microRNAs (miRNAs) profiling to name just a few, providing substantial future growth opportunities for LoC devices [11-18]. LoC approaches could overcome the technical limitations of nucleic acid mass screening by providing rapid, cheap and multiplexed assays $[13,15]$.

Among the advanced biochip-based technologies, STMicroelectronics has developed a disposable silicon-based micro electro mechanical system (MEMS) LoC device as a part of their "In-Check" platform [19-21]. This platform combines all the functions needed to identify given oligonucleotide sequences in a sample and includes microfluidic handling, a miniaturized PCR reactorand a nucleic acid microarray detection module (Figure 1).

The In-Check platform has already been used successfully to amplify human genome sequences and detect human genome mutations, such as the gene associated with $\beta$-thalassemia as well as the detection of viral infectious diseases with full integration of the PCR amplification with subsequent microarray detection [22-24].

Previously, the chemical-based approach for nucleic acid testing (Chem-NAT) commercialised by DestiNA Genomics had been validated by genotyping, with 100\% read accuracy, using DNA from mouth swabs from Cystic Fibrosis (CF) patients and mass spectrometry (MALDI-ToF) for analysis [25].

Briefly, DestiNA core technology takes advantage of dynamic chemistry for nucleic acid sequence specific recognition using aldehyde-modified natural nucleobases (so called SMART nucleobases), and probes based on peptide nucleic acid (PNA), containing an "abasic" position (DestiNA probes) which can be made complementary to any target nucleic acid sequence (Figure 2(A)) [26].

A major feature of Chem-NAT is that false positives are difficult if not impossible to create as nucleobase incorporation can only occur in the presence of target templating nucleic acid strands Figure 2(A).

While mass spectrometry allows single base discrimination and multiplexing capabilities due to molecular weight differences between SMART nucleobases, fluorescence based assays require the DestiNA SMART nucleobases to be fluorescently-labelled and the DestiNA probes to be modified to allow their covalent immobilisation on surfaces Figure 2(B,C). 
Figure 1. The main components of In-Check platform: (A) The Lab-on-Chip core device amplifies clinically relevant DNA samples by Polymerase Chain Reaction (PCR) and has an integrated custom low-density microarray (showed by the white arrow). (B) In-Check platform instruments. The Lab-on-Chip interfaces to the Temperature Control System (TCS) that actuates, monitors, and controls the parameters of the reaction. The TCS unit comprises five control modules with independent thermal protocols and random access capability. Optical signal acquisition is performed on a dedicated portable reader and processed by ST's specialized bioinformatics software. The software package allows users to easily monitor and control reaction processes, analyse the results and automatically generate diagnostic reports.

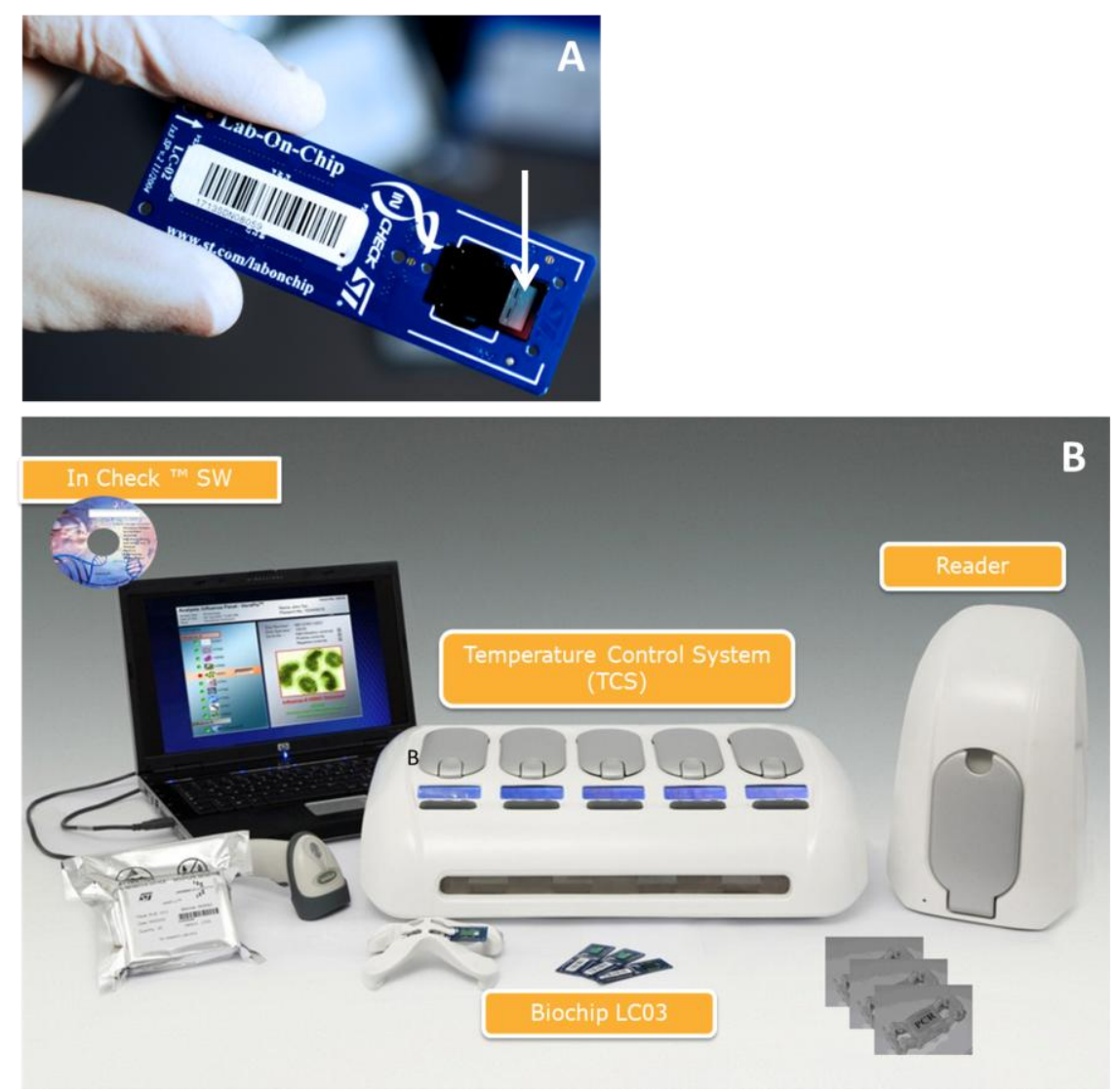

Multiplexing can be achieved by printing probes at defined XY coordinates and by incorporation of the correct fluorescently-labelled SMART nucleobase into the chemical pocket following duplex hybridisation. Such an application allows the use of label-free nucleic acids.

Herein, a proof-of-concept study which integrates DestiNA Genomics Chem-NAT with STMicroelectronics In-Check LoC platform is described, delivering a novel biochip platform for the rapid detection of nucleic acids with high sensitivity and specificity.

The novel biochip platform was evaluated and validated for detection of synthetic small RNAs (sRNAs) based on microRNA-122 (miRNA122) and mengo virus RNA (MGV). This biological model represents the first steps in the development of a novel suite of assays for the medical diagnostic field. Integration of DestiNA technology with the STMicroelectronics In-Check LoC creates a highly innovative product with a true diagnostic potential and utility, for rapid detection of nucleic acids with benefits in terms of result consistency, time, cost, and ease of use. 
Figure 2. (A) The steps involved in DestiNA Genomics chemical-based approach for nucleic acid testing (Chem-NAT). (Copyright Wiley-VCH Verlag GmbH \& Co. KGaA, Reproduced with permission) [26]. DestiNA probe with the target sequence to be detected creates a molecular pocket (step 1) that allows the specific incorporation of a DestiNA SMART nucleobase (step 2) which is then chemically locked into position (step 3). SMART nucleobase incorporation can be directly detected by Mass Spectrometry. (B) Structure of the fluorescein labelled aldehyde Cytosine (FITC- $\mathrm{C}_{\mathrm{CHO}}$ ) for detection using fluorescence reporter system. (C) Structures of a DestiNA probe containing a $\mathrm{N}$-terminated amino peg-ylated spacer for being covalently immobilised onto surfaces.

A
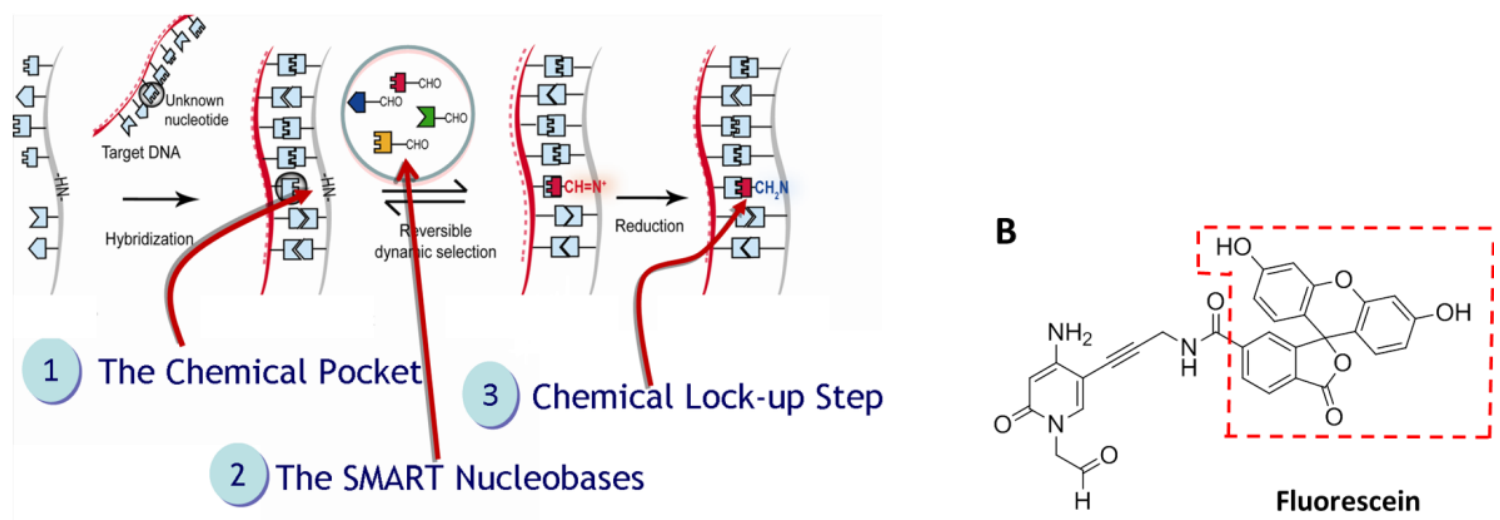

C

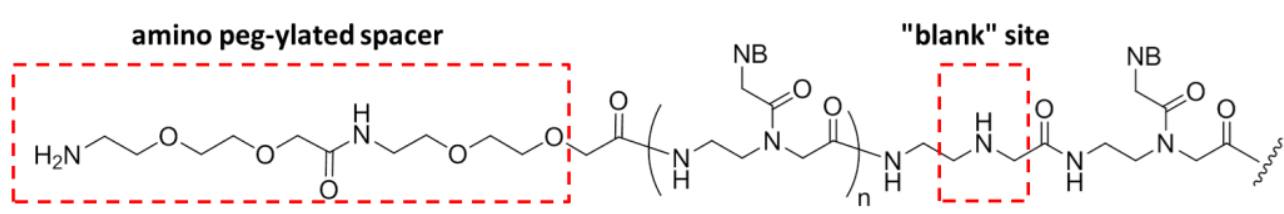

\section{Experimental Procedure and Methods}

\subsection{General}

STMicroelectronics In-Check LoC platforms were fabricated as described previously [19]. Commercially available reagents and buffer for the functionalization of the LoC surfaces were used without further purification. Hydrogen peroxide (29\%), ammonium hydroxide (25\%), hydrochloridric acid (37\%) and methanol were purchased from Sigma Aldrich (Poole,UK) and were used as received. Spot buffer (Nexterion Spot) and hybridisation solution (Nexterion Hyb) were purchased from SCHOTT and were used as received. All synthetic RNA oligomers were purchased in desalted form from Microsynth AG (Balgach, Switzerland).

\subsection{Instrumentations}

A microdrop inject printer equipped with a micro-pipette (AD-K-501 $70 \mu \mathrm{m}$ diameter nozzle) (Microdrop Technologies GmbH, Muehlenweg, Norderstedt, Germany) and a BioAnalyzer 4F/4S equipped with a light scanner (LaVision BioTech $\mathrm{GmbH}$, Bielefeld, Germany) were used. DestiNA probe aqueous solutions concentrations were determined using an Agilent 8453 spectrophotometer. 
Hybridisation was carried out on a Q-Hyb10 Hybridisation Oven Incubation System Deltaspin (Quanta Biotech Ltd., Surrey, UK).

\subsection{Probe Synthesis and Purification}

DestiNA probes terminate with an amino PEG group and were prepared by solid-phase synthesis (SPS) using Fmoc/Bhoc protected monomers as described previously [25] and were characterized by MALDI-TOF mass spectrometry (see Figures S1 and S2 in the Supplementary Information).

\subsection{Synthesis of FITC Labelled Aldehyde Cytosine}

Aldehyde-modified cytosine, tagged with a fluorescein molecule was prepared following the synthetic route described elsewhere [27] and characterized by MALDI-TOF mass spectrometry (see Figure S3 in the Supporting Information).

\subsection{Probes Immobilization}

Attachment of DestiNA probes was carried out according to manufacturer's protocols (SCHOTT). Briefly, amino peg-ylated spacer probes were dissolved in $1 \mathrm{X}$ spotting solution to give $100 \mu \mathrm{M}$ solutions. The solution was spotted onto the epoxysilane-functionalised LoC, followed by incubation under humidity for $30 \mathrm{~min}$ in a hybridisation chamber box (Genetix). Thereafter, the LoCs were washed for $5 \mathrm{~min}$ in $0.1 \%$ Triton $\mathrm{X}-100,2 \times 2 \mathrm{~min}$ in $1 \mathrm{mM} \mathrm{HCl}, 10 \mathrm{~min}$ in $100 \mathrm{mM} \mathrm{KCl}$ and $1 \mathrm{~min}$ in deionized water. After washing, the LoCs were blocked in $150 \mathrm{mM}$ phosphate buffer containing $50 \mathrm{mM}$ dimethylamine, $\mathrm{pH} 9$, for $15 \mathrm{~min}$ at $50{ }^{\circ} \mathrm{C}$ with stirring. The LoCs were washed for one min with deionized water, dried in an oil-free nitrogen stream and stored at room temperature until use.

\subsection{Validation of DestiNA Probes Immobilization}

Features on the LoCs were hybridized with complementary labelled synthetic sRNAs (see Table S1, target 1 and 2, in the Supporting Information). In short, in a $0.2 \mathrm{~mL}$ Eppendorf, $10 \mu \mathrm{L}$ of $100 \mu \mathrm{M}$ of labelled synthetic sRNAs were dissolved in $20 \mu \mathrm{L}$ of $\mathrm{diH}_{2} \mathrm{O}$. Samples were mixed with $30 \mu \mathrm{L}$ of hybridisdation buffer and in accordance with the guidelines provided by STMicrelectronics, this solution was loaded into LoCs (through to the two inlets to reach the microarray area). The arrays were covered with two accessories in plastic (clamps) for array sealing and inserted into the Hybridisation Oven for hybridisation. Hybridisation was standardised by treatment at $50{ }^{\circ} \mathrm{C}$ for $2 \mathrm{~h}$. Thereafter, clamps were removed, and the LoCs were washed for 10 min each in $2 \times \mathrm{SSC}+0.1 \%$ SDS, $2 \times$ SSC and then $0.2 \times$ SSC (all wash steps were performed at room temperature). LoCs were dried using an oil-free nitrogen stream and scanned using a $100 \mathrm{~ms}$ exposure time using emission and detection filters appropriate for FITC.

\subsection{DestiNA Reaction on LoC}

A $0.2 \mathrm{~mL}$ Eppendorf containing $5 \mu \mathrm{L}$ of a $100 \mu \mathrm{M}$ unlabelled miRNA122 oligomer aqueous solution (see Table S1, target 3 in the Supporting Information) was placed in a heater (Techne TC-312 Thermocycler) at $95{ }^{\circ} \mathrm{C}$ for $5 \mathrm{~min}$, then cooled to $40{ }^{\circ} \mathrm{C}$ before being combined with $10 \mu \mathrm{L}$ of 
$\mathrm{NaBH}_{3} \mathrm{CN}(1 \mathrm{M})$ and $15 \mu \mathrm{L}$ of FITC labelled aldehyde cytosine (FITC-C $\mathrm{CHO}_{\text {HO }}(100 \mu \mathrm{M}$ in $0.5 \%$ DMSO aqueous solution). Samples were mixed with $30 \mu \mathrm{L}$ of hybridisation buffer (final volume $60 \mu \mathrm{L}$ ) and loaded onto the LoC as described above. The arrays were covered with the two clamps and placed into the hybridisation oven at $50{ }^{\circ} \mathrm{C}$ for $2 \mathrm{~h}$. After the reaction the LoCs were washed, dried and scanned as described above.

\section{Results and Discussion}

In a first phase of this feasibility study, a protocol to print DestiNA probes onto LoC using an inkjet printer was developed. Once the printing and blocking conditions were optimised, a second phase was carried out in order to check if DestiNA probes, covalently immobilized on the LoC, were able to form duplexes with complementary synthetic sRNA oligomers. In a final stage dynamic incorporation of FITC- $\mathrm{C}_{\mathrm{CHO}}$ was tested to determine if high specificity was achieved on the novel biochip platform.

\subsection{DestiNA Probes for In-Check}

In this study two different RNA strands mimicking natural sRNAs were used to validate the methodologies. The two objects of this study were related to miRNA122 and MGV. miRNA122 is a 22 nucleotide long single strand RNA found in high concentrations in human plasma of patients who have overdosed on paracetamol, becoming a prospective biomarker of liver damage [28]. MGV can be passed to humans through food intake, mainly in shellfish and analysis in the food chain is important to avoid major outbreaks of hepatitis A [29]. The MGV transcript is over 3,000 $\mathrm{Kb}$ long and the target region selected was chosen based on recommendation of the European Committee for Standardisation (CEN/TC 275/WG6/TAG4-viruses in foods).

To investigate and discriminate between the chosen targets, two different DestiNA probes, one to clamp the mature miRNA122 sequence and the one targeting MGV genomic RNA, were designed and synthesised. The design aligned the N-terminal end of the probes with the 3 ' end of their target having the "blank" position lined up opposite to a guanidine residue under interrogation, as shown in Figure 3.

Figure 3. miRNA122 and MGV were interrogated using two different DestiNA probes (1 and 2) 18 and 20 mers respectively. Incorporation of FITC- $\mathrm{C}_{\mathrm{CHO}}$ provides proof-reading, indicating the presence of the complementary oligomers. Only with perfect complementarity the FITC- $\mathrm{C}_{\mathrm{CHO}}$ SMART nucleobase will be incorporated. Probe design was carried out using publicly available database (miRBase Accession Number: MIMAT0000421). MGV was designed using the real-time probe Mengo147 previously published [29]. "*" The single-stranded DNA probe Mengo147 was an identical version of the mengo virus genomic RNA except that "T" bases were replaced with " $U$ " bases.
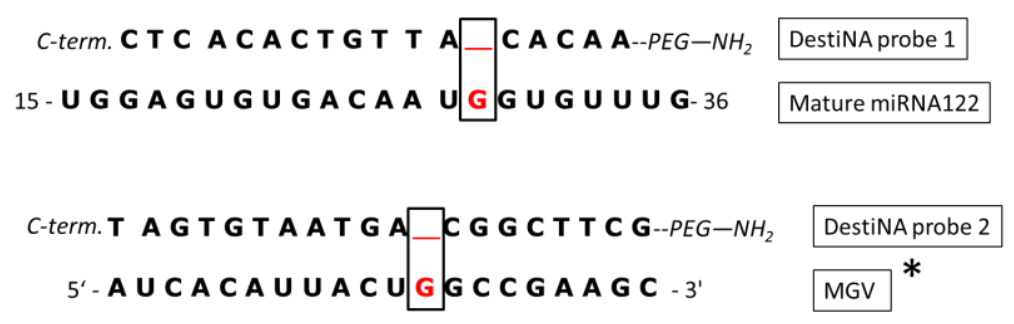
DestiNA probes were reacted chemoselectivity with the epoxy groups that coat the silicon-based microarray surface of the In-Check LoC.

Figure 4 shows an amino-modified DestiNA probe reacting with the epoxysilane surface of the LoC (a reaction that does not need additional baking or UV cross-linking steps). Molecular spacers (PEG group) between the probes and the LoC epoxy groups facilitate interactions between the printed DestiNA probes and their target binding partners in solution.

Figure 4. Modified DestiNA probes immobilization. DestiNA probes were covalently attached through an epoxide ring-opening reaction via their primary amines.
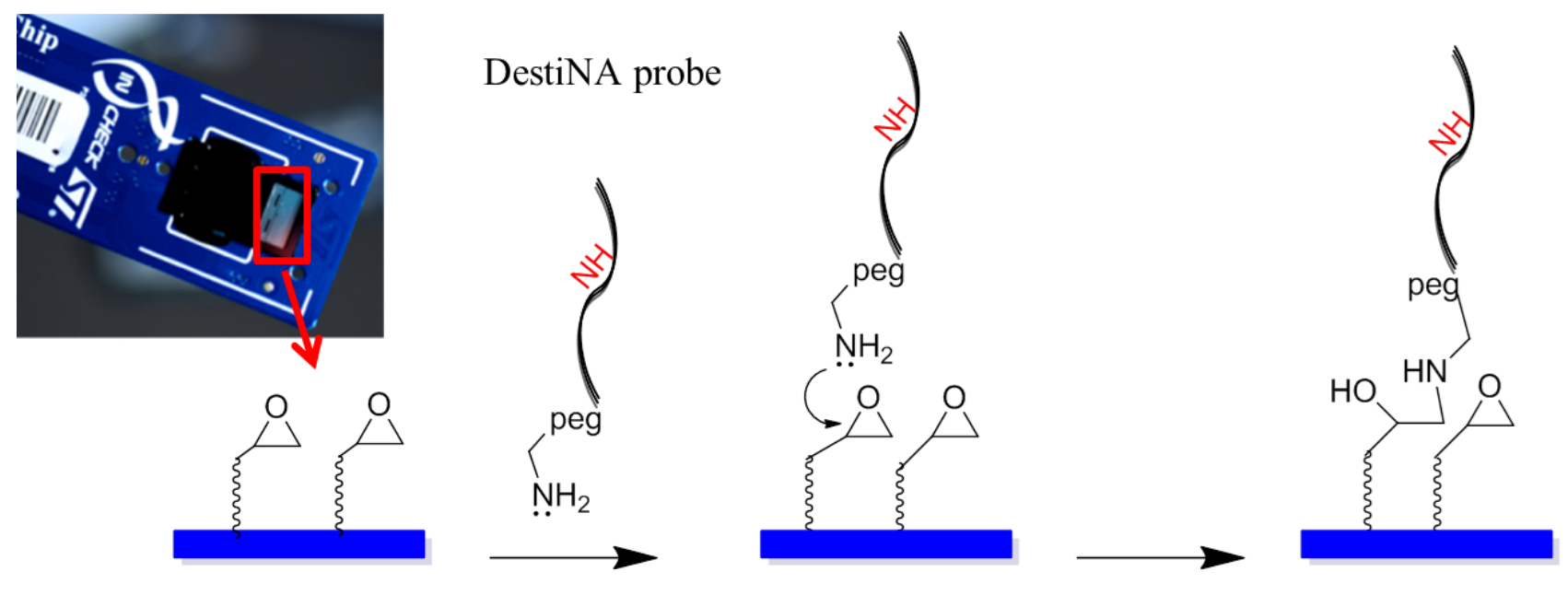

\subsection{Printing and Blocking Optimization}

An important stage in the construction of the novel LoCs was defining the microarray probe layout. The two test probes were printed onto two symmetrical portions of the array (pattern 1 and 2) and arranged in 15 rows and six columns (90 features in total). Taking into consideration the size of the microarray of $3.7 \mathrm{~mm} \times 1.45 \mathrm{~mm}$, it was decided to create a spot pitch of $250 \mu \mathrm{m}$ Figure 5(A). Corner control FITC-labelled DNA oligomers (see Table S1 in the Supporting Information) were printed to identify array orientation. Empty positions to evaluate background noise were provided. Overall, 32 positions were allocated to the control probes and 64 to the specific probes Figure 5(A).

Printing was performed using a proprietary non-contact inkjet printing process in which DestiNA probes were deposited uniformly onto the LoC microarray area. The precise inkjet process enabled the delivery of extremely small, accurate volumes (picoliters) of the chemicals Figure 5(B).

Efficient blocking of reactive surface groups after arraying was critical for a reduced fluorescence background. The LoC was blocked with a $150 \mathrm{mM}$ phosphate buffer containing $50 \mathrm{mM}$ dimethylamine. The tertiary amines engendered prevented reactions with the aldehyde groups of the FITC-C $\mathrm{CHO}_{\mathrm{CHO}}$ Figure 5(C). Standard SCHOTT blocking buffer (containing ethanolamine) blocking approach were excluded as this would give rise to secondary amines on the surface which could react with the aldehyde groups of the fluorescently-labelled SMART nucleobase. 
Figure 5. (A) Graphic layout of the microarray. (B) Printing station with a microdrop printer $\mathrm{GmbH}$ (Norderstedt, Germany) containing a micro-pipette with a $70 \mu \mathrm{m}$ diameter nozzle. (C) Efficient blocking using $150 \mathrm{mM}$ phosphate buffer containing $50 \mathrm{mM}$ dimethylamine. Red square shows the tertiary amines formed.
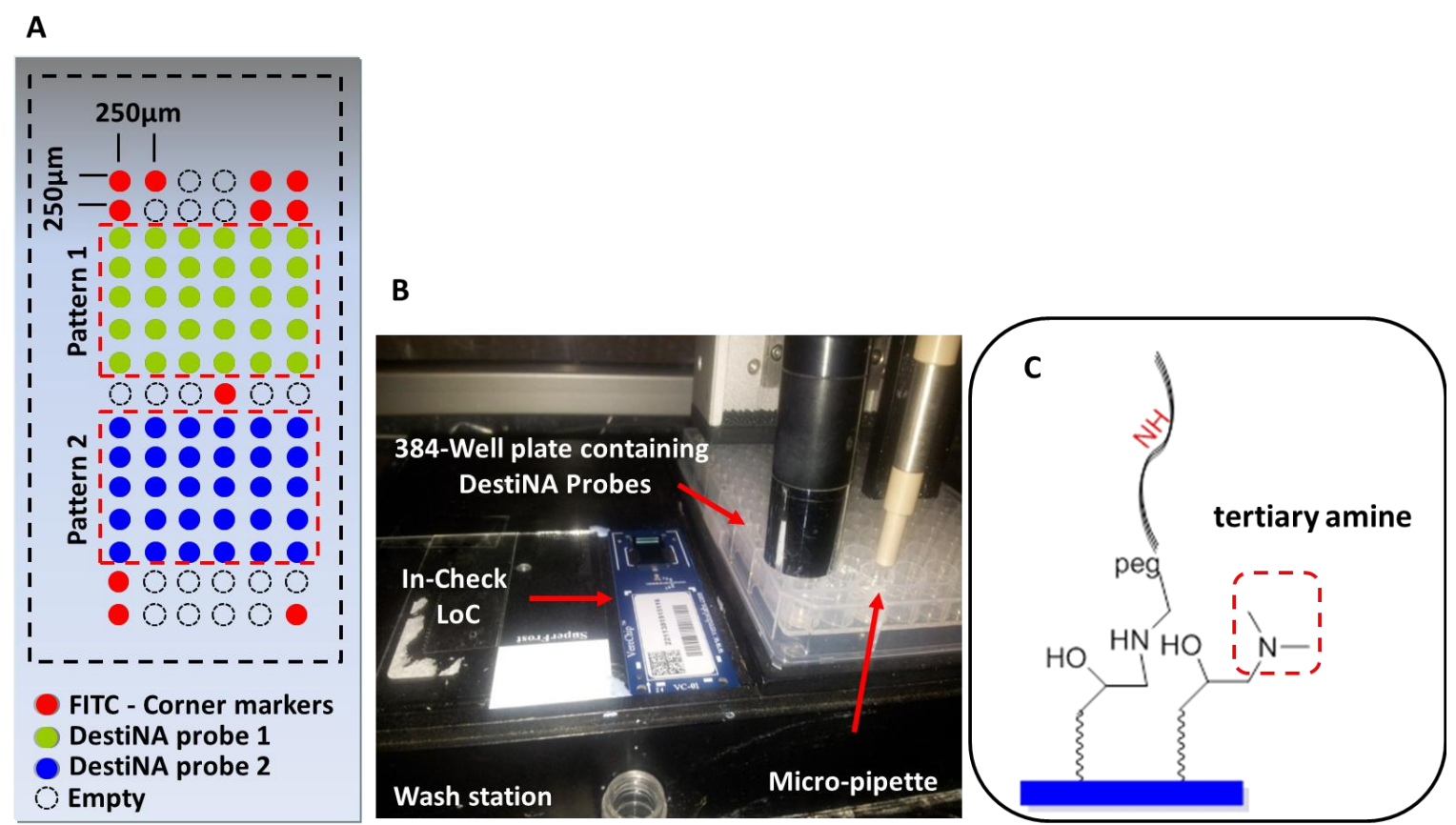

\subsection{Printing Validation with Synthetic Oligonucleotides}

Following printing of the probes on the LoC microarray area, the performance of the DestiNA probes was initially checked by hybridizing complementary fluorescently-labelled sRNA oligomers (see Table S1, target 1 and 2 in the Supporting Information). Figure 6 shows fluorescence scanning images of those areas, clearly demonstrating the ability of the immobilised DestiNA probes to recognise and form duplexes with complementary nucleic acid strands.

Good printing performance and excellent spot morphology for both probe 1 and 2 was observed. Pattern 1 (left image) shows where the oligo miRNA122 FITC plus complementary DestiNA probe 1, attached to the LoC surface, has correctly hybridised and fluoresces. Pattern 2 (left image) shows no fluorescence, as the oligo miRNA122 FITC had no complementarity to DestiNA probe 2 and, was washed away. Additionally, Pattern 2 (right image) shows where the oligo MGV FITC plus complementary DestiNA probe 2 has correctly hybridised and fluoresces, while Pattern 1 (right image) shows no fluorescence, as the oligo MGV had no complementarity to DestiNA probes 1.

\subsection{DestiNA Reaction on In-Check LoC Platform}

After optimising the printing, blocking conditions and validating the capability of DestiNA probes to efficiently hybridise only with complementary sequence sRNA oligomers, the ability to achieve a DestiNA Chem-NAT dynamic chemistry protocol on the LoC devices was explored. Unlabelled miRNA122 (see Table S1, target 3 in the Supporting Information) was used as template strand and FITC- $\mathrm{C}_{\mathrm{CHO}}$ nucleobase was used with LoCs previously arrayed with DestiNA probes (Figure 5(A)). The 
resulting LoC scanning (Figure 7) showed the ability of the DestiNA technology to identify miRNA122 oligomer on In-Check, using a highly selective incorporation of the fluorescently-labelled cytosine.

Figure 6. LoCs scanned after the hybridisation of DestiNA probes with either synthetic miRNA122-FITC (left) or MGV-FITC (right). LoCs were scanned using a $200 \mathrm{~ms}$ exposure time using emission and detection filters appropriate for FITC.
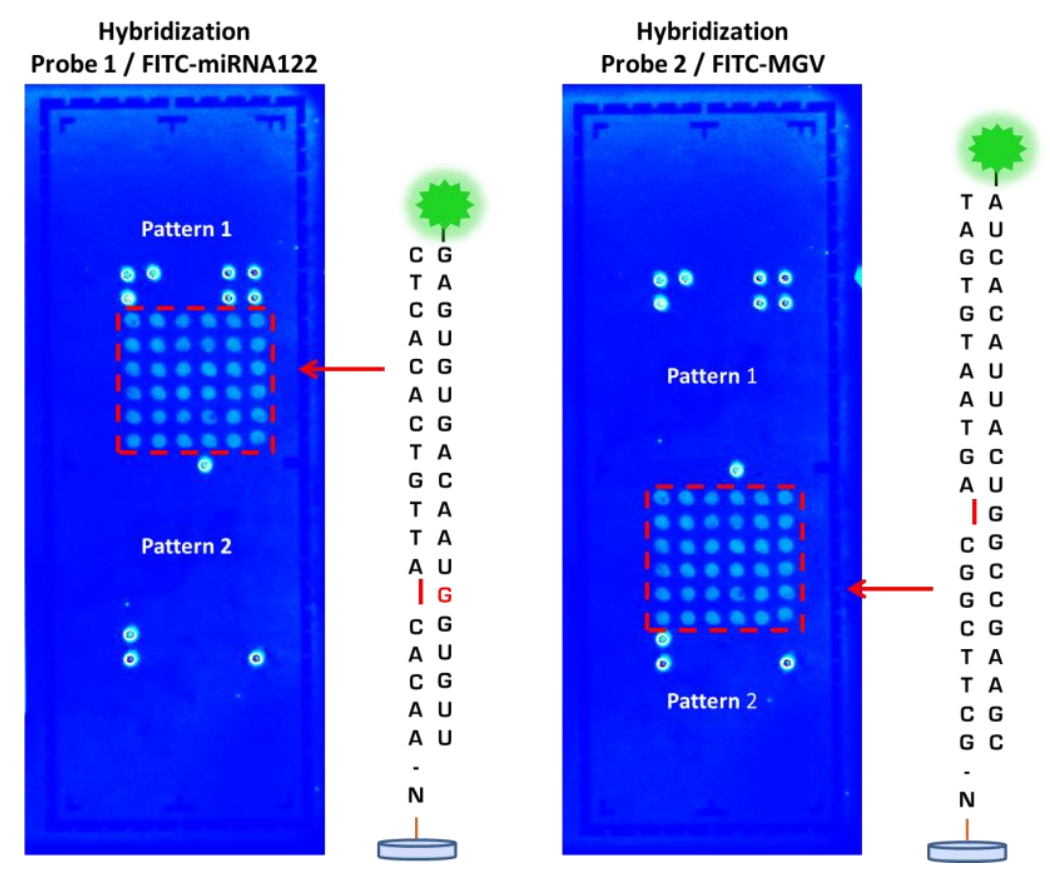

Figure 7. LoC scanned after DestiNA reaction with FITC-C $\mathrm{CHO}_{\mathrm{CHO}}$ Red square (pattern 1) shows where the oligo miRNA122 plus complementary DestiNA probe 1 attached to the array has correctly hybridised and fluorescence due to FITC- $\mathrm{C}_{\mathrm{CHO}}$ incorporation (showed by black arrow). On the other hand, red square (pattern 2) does not show fluorescence due to an absence of hybridisation between oligo miRNA122 plus DestiNA probe 2 (FITC-C $\mathrm{CHO}_{\mathrm{O}}$ incorporation not take place).

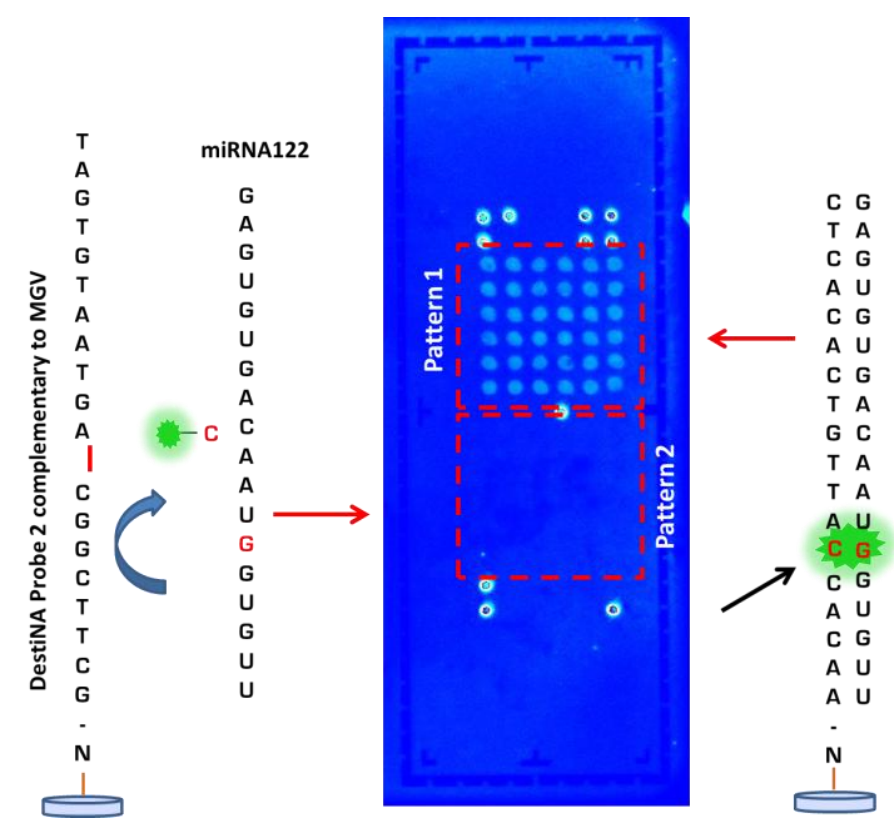


Background was at a very low level, demonstrating excellent blocking and the benefit of the proof reading step using the DestiNA SMART base protocol.

Selective incorporation of FITC- $\mathrm{C}_{\mathrm{CHO}}$ into the PNA/RNA hybrid through dynamic chemical reactions can take place ONLY when miRNA122 forms a perfect duplex with the designed DestiNA probes (pattern 1). Pattern 2 shows no fluorescence because non-complementary (miRNA122) has not been hybridised with the PNA probe 2. This demonstrated the selective incorporation of the "correct" base when the target nucleic acid forms a perfect duplex. The result confirms that the DestiNA fluorescence detection approach to nucleic acid discrimination reported here could be ported into the LoC platform, facilitating the analysis of label-free nucleic acid. The result further indicates that the approach used with miRNA122 herein could be extended to more generic, direct nucleic acid analysis such as allele-discrimination.

\section{Conclusions}

In this manuscript we have given an overview of a "Proof of Concept" study for testing the integration of DestiNA Genomics and STMicroelectronics technologies. All the key proof of concept objectives were achieved, including effective DestiNA probe printing and DestiNA reactions, demonstrating successful translation of DestiNA reagents onto the In Check LoC platform. This study represents a first step in merging the two technologies to create a suite of novel medical diagnostic assays capable of delivering a novel biochip platform for the rapid detection of nucleic acids with high sensitivity and specificity.By combining the novel chemical-based methods with the Lab-on-Chip platform, clinically valuable innovation is achieved through:

1. The unique high affinity and selective hybridisation between DestiNA probes and target DNA or RNA, superior to standard DNA/DNA or DNA/RNA hybridisations.

2. The innovative DestiNA probes, that contain a "blank"/nucleobase free position within the probe, enable a unique proof-reading step to occur. A rapid and selective incorporation of complementary fluorescently labelled SMART nucleobase into the PNA/RNA hybrid can ONLY occur if the target nucleic acid forms a perfect duplex with the designed DestiNA probe.

3. Flexibility to design "personalized" and low cost multiplex assays for target detection in individuals able to be undertaken by laboratory technicians.

The integration and combination of DestiNA Genomics and STMicroelectronics technologies are very promising and potentially suitable for developing a highly innovative next generation system capable of true diagnostic value and utility for the rapid detection of nucleic acids. Benefits for health care providers will be cost benefits in term of reliability, time, and ease of use.

\section{Acknowledgements}

The authors thank F. R. Bowler for his previous work in developing DestiNA technology, and the financial support of Scottish Enterprise for Salvatore Pernagallo and Claudia Cavalluzzo. These studies were approved and supported by DestiNA Genomics and STMicroelectronics. 


\section{References}

1. Hay Burgess, D.C.; Wasserman, J.; Dahl, C.A. Global health diagnostics. Nature 2006, 444, 1-2.

2. Urdea, M.; Penny, L.A.; Olmsted, S.S.; Giovanni, M.Y.; Kaspar, P.; Shepherd, A.; Wilson, P.; Dahl, C.A.; Buchsbaum, S.; Moeller, G.; et al. Requirements for high impact diagnostics in the developing world. Nature 2006, 444, 73-79.

3. Mabey, D.; Peeling, R.W.; Ustianowski, A.; Perkins, M.D. Diagnostics for the developing world. Nat. Rev. Microbiol. 2004, 2, 231-240.

4. Girosi, F.; Olmsted, S.S.; Keeler, E.; Hay Burgess, D.C.; Lim, Y.W.; Aledort, J.E.; Rafael, M.E.; Ricci, K.A.; Boer, R.; Hilborne, L.; et al. Developing and interpreting models to improve diagnostics in developing countries. Nature 2006, 444, 3-8.

5. Houpt, E.R.; Guerrant, R.L. Technology in global health: The need for essential diagnostics. Lancet 2008, 372, 873-874.

6. Smith, C. Tools for drug discovery: Tools of the trade. Nature 2007, 446, 219-222.

7. Miller, M.B.; Tang, Y.-W. Basic concepts of microarrays and potential applications in clinical microbiology. Clin. Microbiol. Rev. 2009, 22, 611-633.

8. Yager, P.; Domingo, G.J.; Gerdes, J. Point-of-care diagnostics for global health. Annu. Rev. Biomed. Eng. 2008, 10, 107-144.

9. Sorger, P.K. Microfluidics closes in on point-of-care assays. Nat. Biotechnol. 2008, 26, 1345-1346.

10. McNerney, R.; Daley, P. Towards a point-of-care test for active tuberculosis: Obstacles and opportunities. Nat. Rev. Microbiol. 2011, 9, 204-213.

11. Horton, B. Nucleic acid testing. Nature 1997, 390, 425-426.

12. Niemz, A.; Ferguson, T.M.; Boyle, D.S. Point-of-care nucleic acid testing for infectious diseases. Trends Biotechnol. 2011, 29, 240-250.

13. Chin, C.D.; Linder, V.; Sia, S.K. Lab-on-a-chip devices for global health: Past studies and future opportunities. Lab Chip 2007, 7, 41-57.

14. Daw, R.; Finkelstein, J. Lab on a chip. Nature 2006, 442, 367.

15. Dutse, S.W.; Yusof, N.A. Microfluidics-based lab-on-chip systems in DNA-based biosensing: An overview. Sensors 2011, 11, 5754-5768.

16. Clarissa, L.; Nathaniel, C.C.; Carl, A.B. Nucleic acid-based detection of bacterial pathogens using integrated microfluidic platform systems. Sensors 2009, 9, 3713-3744.

17. Qian, F.; Baum, M.; Gu, Q.; Morse, D.E. A $1.5 \mathrm{~mL}$ microbial fuel cell for on-chip bioelectricity generation. Lab Chip 2009, 9, 3076-3081.

18. Mairhofer, J.; Roppert, K.; Erlt, P. Microfluidic systems for pathogen sensing. Sensors 2009, 9 , 4804-4823.

19. Palmieri, M.; Alessi, E.; Conoci, S.; Marchi, M.; Panvini, G. Develop the "In-Check" Platform for Diagnostic Applications. In Microfluidics, BioMEMS, and Medical Microsystems VI; Wang, W., Vauchier, C., Eds.; Proceedings of the SPIE: San Jose, CA, USA, 2008; Volume 6886, pp. 688602-1-688602-14.

20. Petralia, S.; Ventimiglia, G. Stability evaluation of protein coating for sensing: An application to silicon based lab-on-chip device. Sens. Transducers J. 2012, 137, 215-225. 
21. Ventimiglia, G.; Petralia, S. Inorganic nanoparticles properties, applications and production photosynthetic routes. Recent Res. Devel. Photochem. Photobiol. 2011, in press.

22. Consolandi, C.; Severgnini, M.; Frosini, A.; Caramenti, G.; de Fazio, M.; Ferrara, F.; Zocco, A.; Fischetti, A.; Palmieri, M.; de Bellis, G. Polymerase chain reaction of 2-kb cyanobacterial gene and human anti- $\alpha_{1}$-chymotrypsin gene from genomic DNA on the In-Check single-use microfabricated silicon chip. Anal. Biochem. 2006, 353, 191-197.

23. Foglieni, B.; Brisci, A.; San Biagio, F.; di Pietro, P.; Petralia, S.; Conoci, S.; Ferrari, M.; Cremonesi, L. Integrated PCR amplification and detection processes on a Lab-on-Chip platform: A new advanced solution for molecular diagnostics. Clin. Chem. Lab. Med. 2010, 48, 329-336.

24. Teo, J.; di Pietro, P.; San Biagio, F.; Capozzoli, M.; Deng, Y.M.; Barr, I.; Caldwell, N.; Ong, K.L.; Sato, M.; Tan, R.; et al. VereFlu ${ }^{\mathrm{TM}}$ : An integrated multiplex RT-PCR and microarray assay for rapid detection and identification of human influenza A and B viruses using lab-on-chip technology. Arch. Virol. 2011, 156, 1371-1378.

25. Bowler, F.R.; Reid, P.A.; Boyd, A.C.; Diaz-Mochon, J.J.; Bradley, M. Dynamic chemistry for enzyme-free allele discrimination in genotyping by MALDI-TOF mass spectrometry. Anal. Methods 2011, 3, 1656-1663.

26. Bowler, F.R.; Diaz-Mochon, J.J.; Swift, M.D.; Bradley, M. DNA Analysis by dynamic chemistry. Angew. Chem. Int. Ed. 2010, 49, 1809-1812.

27. Diaz-Mochon, J.J.; Bradley, M. Nucleobase Characterisation. WO Patent WO2009/037473, 2008.

28. Starkey Lewis, P.J.; Dear, J.; Platt, V.; Simpson, K.J.; Craig, D.G.; Antoine, D.J.; French, N.S.; Dhaun, N.; Webb, D.J.; Costello, E.M.; et al. Circulating microRNAs as potential markers of human drug-induced liver injury. Hepatology 2011, 54, 1767-1776.

29. Pintó, R.M.; Costafreda, M.I.; Bosch, A. Risk assessment in shellfish-borne outbreaks of hepatitis A. Appl. Environ. Microbiol. 2009, 75, 7350-7355.

(C) 2012 by the authors; licensee MDPI, Basel, Switzerland. This article is an open access article distributed under the terms and conditions of the Creative Commons Attribution license (http://creativecommons.org/licenses/by/3.0/). 